\title{
Improving Experiential Learning with Haptic Experimentation
}

\author{
http://dx.doi.org/10.3991/ijoe.v9iS8.3309 \\ L. Santos and C.V. Carvalho \\ GILT-ISEP, Porto, Portugal
}

\begin{abstract}
Experiential learning reflects the idea of knowledge construction as a behavior change resulting from the interaction between the user and the environment leading to experiences, feelings and sensations. The use of virtual simulators in education is a path to foster experiential learning because they provide interactive environments that students can explore and observe. The use of haptic devices with these simulators adds a new dimension, touch, increasing their immersive capacity and the students' attraction and interest. Physics is an area particularly well-suited for the application of haptic simulators. The study of forces, for instance, is an area normally taught in a very abstract and theoretical way and students have extreme difficulty in relating those concepts with their practical application. The "Forces of Physics" haptic simulator described in this article allows experimenting various forces of physics such as the friction force, the aerodynamic force and the gravitational force.
\end{abstract}

Index Terms-Experiential Learning, Haptics, Simulation, Experimentation.

\section{INTRODUCTION}

According to Kolb [1], learning is a behaviour modification as a result of experiences and experiments. Therefore experiential learning values the interaction of the person (experiences and sensations) and the environment (concepts and experiments). Each person, through his/her interpretation of the experience, structures his/her process of knowledge construction.

Virtual simulators are a path to foster experiential learning because they provide interactive environments that students can explore and observe. In fact, a simulation can be defined as the process of designing a model of a real or imaginary system and conducting experiments with that model [2]. Furthermore, the use of virtual simulators enables students to try and perform tasks and operations without the drawbacks of the real environments and without the fear of errors.

But, to create truly immersive environments, there is the need to involve as many natural human senses (hearing, sight, smell, taste and touch) as possible. Common human-machine interaction only includes sight and hearing. Haptic systems represent the addition of touch to this interaction. The term "Haptics" comes from the Greek word haptikós (" $\alpha ँ \pi \omega ")$ and refers to the sense of touch and to the technology, devices, and interfaces that provide tactile feedback [1]. Haptics devices are valued in educational simulations because they provide automatic physical feedback to the user, enabling a more realistic response to a situation.
This article describes the "Forces of Physics" haptic simulator that allows experimenting various forces of physics such as the friction force, the aerodynamic force and the gravitational force.

\section{HAPTIC DEVICES}

Haptic systems are a tactile feedback technology which takes advantage of the sense of touch by applying forces, vibrations or motions to the user. Haptic devices may also incorporate tactile sensors that measure forces exerted by the user on the interface [4]. Initially haptic systems were mainly mechanical, but nowadays other technologies such as ultra-sounds and magnetism allow the user to manipulate virtual elements in a less constrained way with higher degrees of freedom (DOF). A haptic device can have has many as six DOF. Three of those DOF are related to translation: heaving (movement forward/back or back/forward), surging (displacement movement both up and down or the inverse) and swaying (displacement movement either left to right or right to left). The remaining three are related to rotation: pitching (tilting either forward or backward), yawing (swivelling either left or right) and rolling (pivoting side to side).

- Mechanical haptic devices: This type of haptic devices uses mechanical systems like motors and actuators to provide the touch sensation. The most common example of these devices is the Novint Falcon (see Fig. 1), also used in this study.

The Novint Falcon has a single point of force application and three DOF. A much more complex mechanical system is CyberGrasp (see Fig. 2). This device has a structure based on an exoskeleton that allows to perform realistic movement (like grasping virtual objects) because it allows to apply the forces to each finger and to the hand.

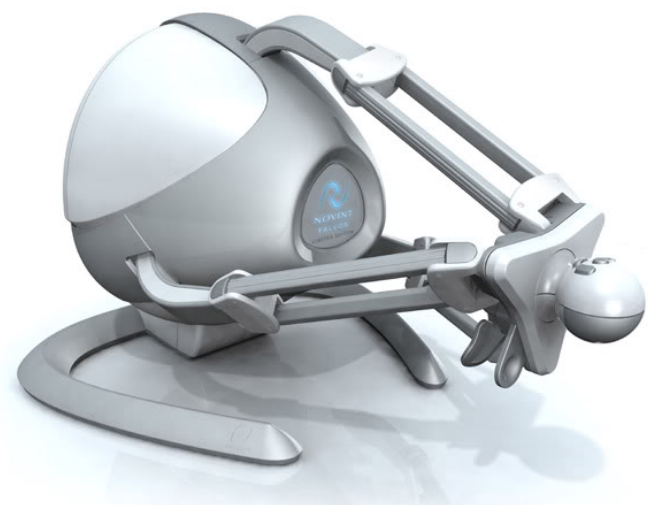

Fig. 1 Novint Falcon mechanical haptic device [5] 
- Magnetic haptic devices: This type of haptic interface uses electromagnetic forces to create the sense of touch. The fact that these devices are wireless allow for a more natural use by the user. A conceptual example of these devices is the ZeroN haptic prototype developed at the MIT Media Lab by Jinha Lee (see Fig. 3). The central element levitates due to the magnetic field and the movement of that metal ball is captured by infrared tracking cameras.

Specialized haptic devices can also be found to perform very specific functions:

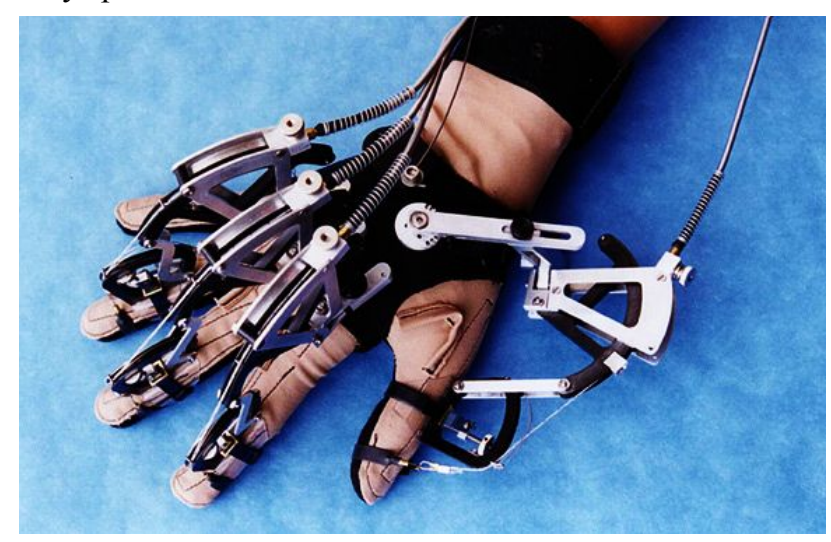

Fig. 2 Cybergrasp mechanical haptic device $[6,7]$

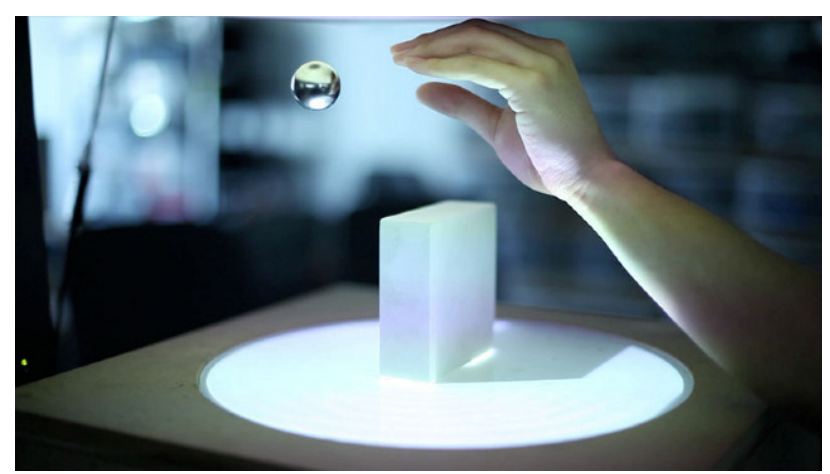

Fig. 3 ZeroN conceptual electromagnetic haptic device [8]

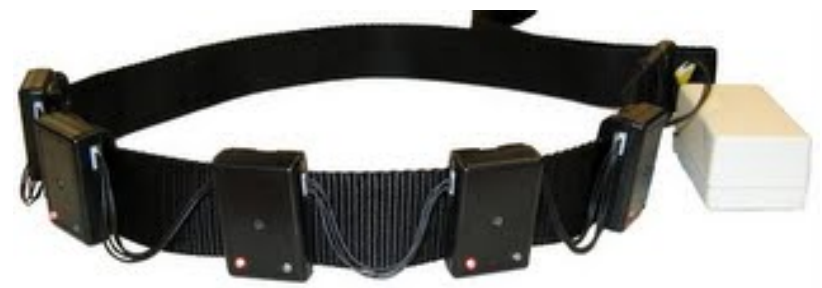

Fig. 4 Haptic belt [9]

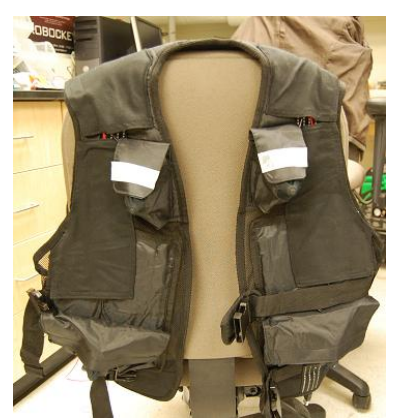

Fig. 5 Haptic vest [10]
- Haptic belt: This type of haptic device was developed by the US Army to guide to the soldiers thanks to a GPS connection and pressure pulses from the belt created by using arrays of vibrating motors (see Fig. 4). Each belts is fitted with eight "tactors" spaced at around 45 degree intervals.

- Haptic vest: The haptic vest concept is similar to the haptic belt, with the "tactors" spread through the inner surface of the vest (see Fig. 5). The vest creates haptic sensations using a solenoid and Peltier elements controlled electronically by a computer.

- Haptic interaction in mobile devices: Haptic interaction in mobile devices is based on the use of patterns of vibration typically used to indicate alarms, incoming calls or other alerts. This type of stimuli is mono-directional and generated across the entire device. In a more advanced approach, piezoelectric actuators may produce non-vibrotactile skin stimulation [11]. "The user places his/her finger on the actuators and the relative movement of the individual piezo tips stretches the skin locally, activating skin mechanoreceptors. Applying specific patterns of distributed skin deformation can create the illusion of touching small-scale shapes and textures" $[12]$.

\section{FORCES OF PHYSICS SIMULATOR}

The virtual haptic simulator presented in this article is divided in three modules, addressing the concepts of specific type of forces: aerodynamics, friction and gravitation.

- The aerodynamics module allows the user to sense the aerodynamics forces applied to an airplane: thrust, drag, lift and weight.

- The friction module enables the user to feel the friction between a cube and the floor. Depending on the materials applied to the cube and to the floor surface and the corresponding static and coefficients of friction, the user will feel more or less resistance to the movement (see Fig. 6).

- In the gravitation module the user interacts with a 3D model representing the earth to divert asteroids. Gravitational forces are applied to the earth and to the asteroid and, depending on the proximity between the earth and the asteroid, the asteroid's trajectory will change and the user will feel the corresponding force.

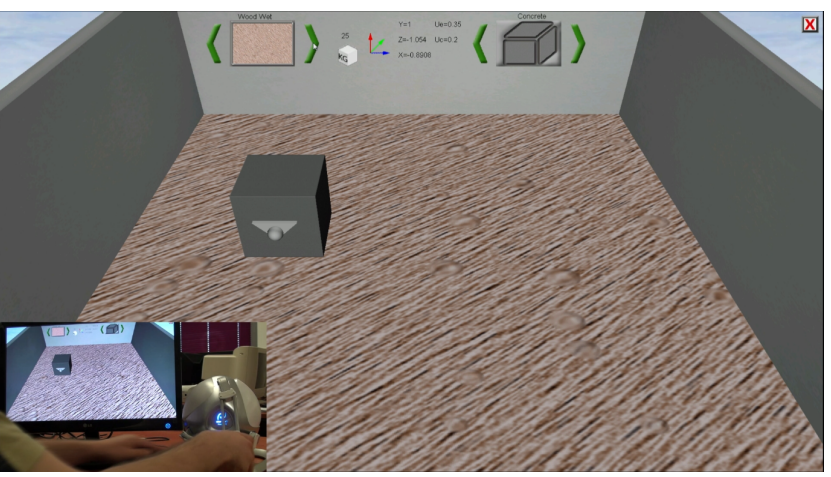

Fig. 6 Friction module of the Forces of Physics simulator 
The simulator was developed with DirectX 9, C++, the Novint Haptic Device Abstraction Layer (HDAL) and the Novint Falcon as hardware. HDAL is a low level API that enables the communication between the Falcon and the computer through functions and resources. The basic accessible information of the Falcon is related with the cursor position and button states but it also enables the programmer to update the force applied unto the device.

The simulator was evaluated in a two-stage procedure:

- Firstly, a group of students used the simulator with the help of a set of laboratory experiments guides. Their pre and post-knowledge was assessed. In general their answers revealed improvements in their level of knowledge. Students were also very positive on the interest and motivation generated by this type of simulations.

- Secondly, a group of experts in haptics and simulation tested the simulator. Semi-structured interviews followed and their comments and observations were registered. The majority of these experts were very positive about the simulator itself $(65 \%)$ and about the use of these environments $(60 \%)$ for learning, therefore corroborating [13].

\section{CONCLUSION}

This article focuses the use of haptic technologies and virtual simulators in education and, particularly, in experiential learning of physics. Haptics devices are very relevant in educational simulations because they provide automatic physical feedback to the user, enabling a more realistic response to a situation.

A haptic simulator called Forces of Physics was designed and developed to help learners understand the relation between the theoretical concepts and their practical application. At this stage this simulator is intended to complement the more traditional educational process by providing the students with hands-on experiences.

The first evaluation results, with students and experts in simulations and haptics, provided motivating data in view of future developments of the simulator.

\section{REFERENCES}

[1] Kolb, D. Experiential Learning. New Jersey: Prentice-Hall. 1984

[2] Ralstone A. and Reily E.D., Encyclopedia of Computer Science and Engineering, van Nostrand, New York, 1983
[3] Srinivasan, M A and Basdogan, C, "Haptics in Virtual Environments: Taxonomy, Research Status and Challenges", Computers and Graphics, Vol. 21, No. 4, 1997. http://dx.doi.org/10.1016/S0097-8493(97)00030-7

[4] Robles-De-La-Torre G. "Virtual Reality: Touch / Haptics". Sage Encyclopedia of Perception. Goldstein B (Ed.), Sage Publications, Thousand Oaks CA, 2009.

[5] Novint, Novint Falcon, [Online] Available at http://www.novint.com/index.php/novintfalcon

[6] CyberGlove, CyberGrasp, [Online] Available at http://www.cyberglovesystems.com/products/cybergrasp/overview

[7] R. Leuschke, E. K. Kurihara, and J., \& Hannaford, B. Dosher, "High fidelity multi finger haptic display," in Proceedings of the First Joint Eurohaptics Conference and Symposium on Haptic Interfaces for Virtual Environment and Teleoperator Systems. World Haptics 2, 2005, pp. 606-608.

[8] Lee, J. and Post, R. Levitated Interaction Element, [Online] Available at http://leejinha.com/ZeroN

[9] Geeky Gadgets, US Army Testing Haptic Belts To Guide Soldiers On The Battlefield, [Online] Available at http://www.geekygadgets.com/us-army-testing-haptic-belts-to-guide-soldiers-onthe-battlefield-30-06-2011/

[10] Palan S., Tactile Gaming Vest, [Online] Available at http://iroboticist.com/2010/03/26/tgv/

[11] Hayward, V. and Cruz-Hernandez, M. "Tactile display device using distributed lateral skin stretch". In Proc. Haptic Interfaces for Virtual Environment and Teleoperator Sys. Symposium, IEEE, pp. 1309-1314, 2000

[12] Luk J., Pasquero J., Little S., MacLean K., Lévesque V. and Hayward V. "A Role for Haptics in Mobile Interaction: Initial Design Using a Handheld Tactile Display Prototype", Proceedings of the CHI 2006, Montréal, Québec, Canada, 2006

[13] A. M., Richard, C., and Cutkosky, M. Okamura, "Feeling is believing: Using a force-feedback joystick to teach dynamic systems," Journal of Engineering Education, 91(3), pp. 345-349, 2002. http://dx.doi.org/10.1002/j.2168-9830.2002.tb00713.x

\section{AUTHORS}

L. Santos is with Graphics, Interaction and Learning Technologies R\&D Group at the Instituto Superior de Engenharia do Porto (e-mail: 1fons@isep.ipp.pt).

C.V. Carvalho is the director of the Graphics, Interaction and Learning Technologies R\&D Group at the Instituto Superior de Engenharia do Porto (e-mail: lfons@isep.ipp.pt).

This article is an extended and modified version of a paper presented at the International Conference exp.at'13, held 18-20 September 2013, in Coimbra, Portugal. This work was supported in part by the European Comission, through the ALFA Gaviota project. Submitted, November 03, 2013. Published as re-submitted by the authors 04 December 2013. 